Intensitätsverlusten durch die IR-Absorption von $\mathrm{CO}_{2}$ und Wasserdampf wurde bei den Aufnahmen im CsBrBereich durch Spülen mit gereinigtem Stickstoff eine feuchtigkeits- und $\mathrm{CO}_{2}$-freie Atmosphäre im Gerät aufrecht erhalten. Einzelne Proben der Wasserstoffverbindungen wurden zum Vergleich auch als KBr-Preßlinge vermessen, wobei keine Änderungen gegenüber den Nujol-Spektren zu beobachten waren.

18 Brauer, Handbuch der präparativen anorganischen Chemie, F. Enke Verlag Stuttgart, 1962.
Herrn Professor Dr. Dr. h. c. W. Hieber danken wir für die Förderung unserer Arbeit auf das herzlichste; ebenso danken wir Herrn Professor Dr. H. Zimmermann für wertvolle Diskussionen. Der D e u t s chen F o r schungsgemeinschaft und dem Verband der Chemischen Industrie sind wir für die gewährte Unterstützung zu großem Dank verpflichtet.

19 Vgl. F. Hein u. H. Lilie, Z. anorg. allg. Chem. 270, 45 [1952].

\title{
Synthese von Serinpeptiden I
}

\author{
H. Voss \\ Institut für Lebensmittelchemie und Lebensmitteltechnologie, Technische Universität Berlin \\ (Z. Naturforschg. 20 b, 116-121 [1965] ; eingegangen am 15. Oktober 1964)
}

\begin{abstract}
Es wird über die Synthese einer Reihe von $N$-Carbobenzoxy-aminosäuren und -peptidsäuren sowie Aminosäure- und Peptidbenzylester-Derivaten, die als Ausgangsprodukte zur Darstellung verschiedener Serinpeptide dienten, berichtet.
\end{abstract}

(Die verwendeten Abkürzungen: Aminosäuresymbole nach E. Brand u. J. T. Edsall, Ann. Rev. Biochem. 16, 223 [1947]; DCC = Dicyclohexylcarbodiimid; DMF $=$ Dimethylformamid; $\mathrm{OBz}=$ Benzylester; $\mathrm{pts}=p$-Toluolsulfonsäure; $\mathrm{Z}=$ Carbobenzoxy.)

Im Rahmen von enzymatischen Untersuchungen an Casein- und Eidotterprodukten waren an unserem Institut Peptidgemische erhalten worden, die u. a. phosphorsäurehaltige Serinpeptide enthielten. Solche Serinpeptide scheinen eine besondere physiologische Wirkung zu besitzen. Aus diesem Grunde sollten sie näher untersucht werden. Eine Isolierung der einzelnen Peptide aus dem Gemisch bereitete jedoch große Schwierigkeiten. Säulenchromatographisch waren verschiedene Fraktionen erhalten worden, die sich aber nicht weiter auftrennen ließen, wie es für eine Sequenzanalyse der einzelnen Komponenten des Gemisches erforderlich war. Daher wurde zunächst durch. Totalhydrolyse und papierchromatische Auftrennung das Spektrum der in den verschiedenen Fraktionen enthaltenden Aminosäuren ermittelt. An Hand der hiermit ermittelten einzelnen Aminosäuren sollten nun mittels Modellsubstanzen neue Erkenntnisse über die Eigenschaften derartiger Serinpeptide, und zwar sowohl in phosphorylierter als auch in nicht

1 E. Fischer, Ber. dtsch. chem. Ges. 40, 1501 [1907].

2 E. Fischer u. U. Suzuki, Ber. dtsch. chem. Ges. 38, 4173 [1905].

3 J. S. Fruton, J. biol. Chemistry 146, 463 [1942]. phosphorylierter Form, gewonnen werden, die für die weiteren Auftrennungen und Untersuchungen nutzbringend verwendet werden konnten.

In der vorliegenden Arbeit wird über die Synthese von phosphorsäurefreien Di-, Tri-, Tetra- und Pentapeptiden des Serins nebst den dazugehörigen Ausgangsderivaten berichtet.

Serinpeptide wurden erstmals von Fischer ${ }^{1,2}$ in Form des racemischen H-DL-Ser-DL-ser-OH und eines teilweise racemisierten optisch aktiven H-L-Ser-L-ser$\mathrm{OH}$ synthetisiert. Aber erst Fruton ${ }^{3,4}$ war es, der mit Hilfe der Azidmethode eine größere Anzahl von optisch aktiven Serinpeptiden ohne Maskierung der Hydroxylgruppe des Serins, darstellte. Unter Verwendung von DCC als Peptid-Kondensationsmittel bereitete später FöLsch ${ }^{5}$ eine weitere Anzahl optisch aktiver Serinpeptide. An diese zuletzt erwähnten Veröffentlichungen wurde in der vorliegenden Arbeit im wesentlichen angeknüpft.

Das zu lösende Problem führte zu einer speziellen Aufgabenstellung: Es waren eine Reihe von optisch aktiven Serinpeptiden mit bestimmter Aminosäuresequenz zu synthetisieren. Die Hydroxylgruppe des Serins hatte dabei ungeschützt zu bleiben, um später-

${ }^{4}$ J. I. Harris u. J. S. Fruton, J. biol. Chemistry 191, 143 [1951].

5 G. FöLsch, Acta chem. scand. 13, 1407 [1959]. 
hin weitere Reaktionen, insbesondere eine Phosphorylierung, zu ermöglichen. Diese Peptide waren an den terminalen Amino- und Carboxylgruppen mit solchen Schutzgruppen zu versehen, die sich leicht wieder abspalten ließen und bei deren Abspaltung eine vorhandene $\mathrm{O}-\mathrm{P}$-Esterbindung an der Hydroxylgruppe des Serins erhalten blieb. Die Synthesewege waren so auszuwählen und auszuarbeiten, daß keine bzw. nur geringe Racemisierung erfolgte und die Möglichkeit gegeben war, die einzelnen Peptide in präparativ größerem Maßstab darzustellen. Das bedeutete, daß vom Endprodukt, entsprechend der Aufgabenstellung, alle Schutzgruppen mit einer einzigen schonenden Operation entfernt werden sollten. Diese Möglichkeit ließ sich durch die katalytische Abhydrierung der einzelnen Schutzgruppen erreichen. Durch die Festlegung dieses Syntheseweges wurde die große Zahl der vielen bekannten Schutzgruppen auf einige wenige eingeschränkt. Zum Schutz der Aminogruppe kamen nur noch die Carbobenzoxyund die Triphenylmethylgruppe und für die Carboxylgruppe die Benzyl- bzw. p-Nitrobenzylester in Frage. Von diesen vier Schutzgruppen wurden die Carbobenzoxygruppe und die Benzylestergruppierung für die Synthese ausgewählt.

Zur Herstellung der gewünschten $N$-Carbobenzoxyaminosäuren und -peptidsäuren wurde ein Verfahren von Gutrmann und Borssonnas ${ }^{6}$ angewandt, welches bisher nur zur Bereitung des $N$-Z-L-Ser-OH angegeben wurde. Es zeigte sich, daß sich auch andere Aminosäuren und das Peptid Glycyl-glycin mit gutem Erfolg nach dieser Methode carbobenzoxylieren ließen. Die Ausbeuten lagen zwischen $65-89 \%$ d. Theorie. Eine Schwierigkeit, reine, kristallisierte Produkte zu erhalten, trat insofern auf, als beim Ansäuern der Lösung z. T. Komplexbildung eintrat. Es bilden sich Gemische der $N$-Carbobenzoxy-aminosäuren mit ihren Salzen ${ }^{7-10}$, die ebenfalls in organischen Lösungsmitteln löslich sind. Die Komplexbildung konnte teilweise dadurch erkannt werden, daß zwei Produkte mit verschiedenen Schmelzpunkten erhalten wurden, z. B. $N$-Z-L-Phe-OH, oder daß z. B. der $N$-Z-L- $\alpha$-Ala-

6 S. Guttmann u. R. A. Boissonnas, Helv. chim. Acta 41, 1852 [1958].

7 S. S. Brown u. R. Wade, J. chem. Soc. [London] 1962 , 3280 .

8 M. Goodman u. K. C. Stueben, J. org. Chemistry 24, 112 [1959].

9 W. Grassmann u. E. Wünsch, Chem. Ber. 91, 462 [1958].

10 E. P. Grommers u. J. F. Arens, Rec. trav. chim. Pays-Bas 78, 558 [1959].
OH-Komplex ein Öl darstellte, während das reine $N$-Z-L- $\alpha$-Ala-OH kristallin war. Durch starkes Ansäuern und durch nachträgliches Waschen der im organischen Lösungsmittel gelösten Produkte mit 0,5-n. bzw. 1-n. HCl konnte diese Schwierigkeit beseitigt werden.

Durch Verseifung von $N$-Carbobenzoxy-peptidbenzylestern mit $1-n$. $\mathrm{NaOH}$ wurden die entsprechenden $N$-Carbobenzoxy-peptid-säuren erhalten. Während bei serinfreien $N$-Carbobenzoxy-benzylestern die Verseifung ohne Schwierigkeiten in ein bis zwei Stdn. mit guten Ausbeuten erfolgte, waren für die Verseifung serinhaltiger $N$-Carbobenzoxy-peptidbenzylester oftmals mehrere Tage notwendig, ehe eine weitgehende Verseifung eingetreten war. Die erzielten Ausbeuten lagen zwischen $30-70 \%$ d. Theorie. Schon früher machten Harris und Fruton ${ }^{4}$ die Beobachtung, da $\beta$ bei der Verseifung von serinhaltigen $N$-Carbobenzoxy-peptidestern Nebenreaktionen auftraten. Die Carbobenzoxygruppe ist bei der Verseifung von $N$-Carbobenzoxy-peptidestern nicht vollkommen alkalibeständig und bildet als Nebenprodukte Harnstoff- bzw. Hydantoin-Derivate ${ }^{11}, 12,13$. Außerdem besteht die Möglichkeit der Peptidspaltung, die bei der Verseifung von Methylund Äthylestern von Serinpeptiden beobachtet wurde $^{4,6}$. Die Verseifung mußte auch unter sehr schonenden Bedingungen erfolgen, da Serin eine der am leichtesten racemisierenden Aminosäure ${ }^{14}$ ist und eine Racemisierung im alkalischen Medium bevorzugt erfolgt. Aus diesem Grunde wurden die Carbobenzoxy-peptidbenzylester mit einem Unterschuß oder gerade der theoretisch notwendigen Menge an $\mathrm{NaOH}$ bei Raumtemperatur stehen gelassen und danach aufgearbeitet. Die Abtrennung von unverseiftem $N$-Carbobenzoxy-peptidbenzylester erfolgte dabei nach dem Ansäuern der natronalkalischen Lösung mit $\mathrm{HCl}$ und Extraktion mit einem organischen Lösungsmittel durch erneute Extraktion oder Lösung mit 1-m. Kaliumhydrogencarbonat, worin sich nur die $N$-Carbobenzoxy-peptidsäuren und ein Teil der Verunreinigungen lösten. Diese Kalium-

11 W. Grassmann, E. Wünsch u. G. Fürst, G. Fürst: Dipl.Arbeit, Univ. München 1955; E. Wünsch: Dissert., Univ. München 1955.

12 K. Schlögl u. H. Fabitschowitz, Mh. Chem. 84, 937 [1953].

13 S. G. W $W_{\text {aley }}$ u. J. Warson, J. chem. Soc. [London] 1953, 475 .

14 F. S. Daft u. R. D. Coghill, J. biol. Chemistry 99, 213 [1931]. 
hydrogencarbonat-Lösung wurde darauf mit $\mathrm{HCl}$ angesäuert, die $N$-Carbobenzoxy-peptidsäure mit einem organischen Lösungsmittel extrahiert und nach Trocknung und Abdestillation des Lösungsmittels umkristallisiert. Als einzigste der dargestellten Verbindungen war das $N$-Z-L-Ser-L-leu-OH nicht zur Kristallisation zu bringen und mußte daher als Rohprodukt bei den späteren Reaktionen eingesetzt werden. Alle anderen Produkte konnten in kristallisierter Form erhalten werden.

Nach einem Verfahren von Zervas und Mitarbb. ${ }^{15}$ wurden die meisten Aminosäurebenzylester$p$-toluolsulfonate in 70-90-proz. Ausbeute analysenrein hergestellt. H-DL-Ser-OBz-pts und $\mathrm{H}-\mathrm{L}-\mathrm{Ser}-\mathrm{OBz}$ pts wurden nach einer von $\mathrm{FöLSCH}^{5}$ angegebenen Methode gewonnen. H-DL-Ser-OBz ist bisher nur als Benzolsulfonat und als Hydrochlorid ${ }^{16,17}$ dargestellt worden, aber noch nicht als $p$-Toluolsulfonat.

Peptide lassen sich leichter zu den entsprechenden Benzylestern verestern. Ein von Crofts und Mitarbb. ${ }^{18}$ angegebenes Verfahren wurde für die Darstellung einer Reihe bisher nicht auf diese Weise veresterter Peptide anzuwenden versucht. Bei diesen Versuchen konnten eine Anzahl neuer Peptidbenzylester-p-toluolsulfonate gewonnen werden. Sogar die Veresterung des Tetrapeptides Tetraglycin gelang auf diese Art. Bis auf zwei Ausnahmen waren alle neu dargestellten Produkte kristallin. Peptide, die das Serin C-terminal enthielten wie Glycyl-L-serin und Glycyl-glycyl-L-serin, ließen sich nicht kristallisiert erhalten und mußten in Form der mehrmals umgefällten Rohprodukte für die weiteren Reaktionen eingesetzt werden. Dabei zeigte es sich später bei dem Versuch, das geschützte Pentapeptid $N$. Carbobenzoxy-tetraglycyl-L-serinbenzylester herzustellen, daß eine Veresterung des Tripeptides Diglycyl-L-serin auf diese Weise nicht erfolgt war. Das gewünschte Peptid ließ sich nicht isolieren. Aus diesem Grunde wurde ein anderer Weg eingeschlagen. Durch Abspaltung des Carbobenzoxyrestes mit $\mathrm{HBr}$ kann man ebenfalls zu Peptidester-Derivaten gelan-

15 L. Zervas, M. Winitz u. J. P. Greenstein, J. org. Chemistry 22, 1515 [1957].

16 G. Riley, T. H. Turnbull u. W. Wilson, J. chem. Soc. [London] 1957, 1373.

17 H. ZahN u. J. F. Diehl, Z. Naturforschg. 12 b, 85 [1957].

18 P. C. Crofts, J. H. H. Markes u. H. N. Rydon, J. chem. Soc. [London] 1959, 3610.

19 D. Ben-Ishai u. A. Berger, J. org. Chemistry 17, 1564 [1952].

20 S. Guttmann u. R. A. Boissonnas, Helv. chim. Acta 42, 1257 [1959]. gen. Zunächst wurde hierfür ein Verfahren von BENIshaI und Berger ${ }^{19}$ verwandt. Sie arbeiteten mit einer Lösung von $\mathrm{HBr}$ in Eisessig. Das Verfahren besitzt aber verschiedene Nachteile. Enthält das betreffende Peptid Serin, so kann $O$-Acetylierung ${ }^{6,20,21}$ erfolgen und außerdem kann eine teilweise Verseifung der Estergruppen ${ }^{22,} 23$ eintreten. Dieses zeigte sich, als $N$-Z-DL-Ser-gly-OBz mit einer bei $-10{ }^{\circ} \mathrm{C}$ gesättigten Lösung von $\mathrm{HBr}$ in Eisessig umgesetzt wurde. Es wurde lediglich ein uneinheitliches Produkt in Form einer Schmiere erhalten. Durch weitere Umsetzung mit $N$-Z-Gly-OH wurde deshalb versucht, das geschützte Tripeptid $N$-Z-Gly-DL-ser-gly$\mathrm{OBz}$ aufzubauen. Der Versuch mißlang; das geschützte Tripeptid konnte nicht isoliert werden. Dagegen führte die Einleitung von gasförmigem $\mathrm{HBr}$ in eine Suspension des geschützten Serinpeptides in Nitromethan zu dem gewünschten Erfolg. Hierbei wurde in Anlehnung einer von Benorton und Rydon ${ }^{24}$ angegebenen Vorschrift gearbeitet. Das geschützte Tripeptid $N$-Z-Gly-gly-L-ser-OBz ließ sich auf diese Weise in guter Ausbeute in das kristallisierte Tripeptidbenzylester-hydrobromid überführen. Nach mehrmaligem Umkristallisieren ergab die Elementaranalyse ein nicht ganz analysenreines Produkt. Der Aufbau höherer geschützter Serinpeptide verlief mit diesem Produkt erfolgreich.

In den nachstehenden Tabellen wurden die einzelnen Verbindungen zusammengefaßt und mit Literaturhinweisen versehen, soweit sie bereits früher schon einmal, wenn z. T. auch nach anderen Methoden, synthetisiert worden waren.

\section{Experimenteller Teil}

Alle Schmelzpunkte sind unkorrigiert. Sie wurden auf dem Mikroskopheiztisch 350 der Firma E. Leitz $\mathrm{GmbH}$, Wetzlar, bestimmt.

Die Elementar-Analysen wurden im Organisch-Chemischen Institut der Technischen Universität Berlin ausgeführt *

${ }^{21}$ S. Gutrmann u. R. A. Borssonnas, Experientia [Basel] 17, 265 [1961].

22 J. Bayer, S. Dualszky u. L. Kisfaludy, J. Chromatogr. [Amsterdam] 6, 155 [1961].

${ }^{23}$ L. Kisfaludy, S. Dualszky u. J. Bayer, Chimia 14, 368 [1960].

24 L. Benorton u. H. N. Rydos, J. chem. Soc. [London] 1960, 3328.

* Fräulein Dr. U. FaAss bin ich für die Durchführung der Analysen zu Dank verpflichtet. 


\begin{tabular}{|c|c|c|c|c|c|}
\hline Verbindung & $\begin{array}{l}\text { Schmp. } \\
{\left[{ }^{\circ} \mathrm{C}\right]}\end{array}$ & Literatur & $\begin{array}{c}\text { Lit.-Schmp. } \\
{\left[{ }^{\circ} \mathrm{C}\right]}\end{array}$ & $\begin{array}{c}\text { Ausbeute } \\
{[\%]}\end{array}$ & Umkrist. aus \\
\hline$N$-Z-L- $\alpha$-Ala-OH & $86-88$ & 10,33 & $84-86$ & 65,2 & $\mathrm{Ee}$ \\
\hline $\mathrm{N}$-Z-Gly-OH & $122,5-123,5$ & $\begin{array}{c}10 \\
34,35 \\
36\end{array}$ & $\begin{array}{c}119-120 \\
120 \\
122\end{array}$ & 79,8 & Chl \\
\hline$N$-Z-L-Leu-OH & Öl & 9 & Öl & - & - \\
\hline$N$-Z-DL-Ser-OH & $127,5-129,5$ & 34 & 125 & 82,4 & $\mathrm{Ee} / \mathrm{Chl}$ \\
\hline$N$-Z-L-Ser-OH & $120-121$ & $\begin{array}{r}3 \\
6 \\
37 \\
38\end{array}$ & $\begin{array}{c}121 \\
119,5 \\
119-120 \\
117-119\end{array}$ & 81,8 & $\mathrm{Ee} / \mathrm{Chl}$ \\
\hline$N$-Z-Gly-gly-OH & $183,5-185,5$ & $27,34,39$ & 178 & 89,0 & $\mathrm{H}_{2} \mathrm{O}$ \\
\hline$N$-Z-L- $\alpha$-Ala-L-phe-OH & $130-132$ & $\begin{array}{l}25 \\
26\end{array}$ & $\begin{array}{c}56-58 \\
122\end{array}$ & 82,9 & $\mathrm{Ee} / \mathrm{PAe}$ \\
\hline$N$-Z-L-Leu-gly-OH & $119-121$ & $\begin{array}{l}27 \\
28 \\
29 \\
30 \\
31\end{array}$ & $\begin{array}{c}118 \\
117 \\
114-115 \\
112-113 \\
113\end{array}$ & 90,8 & $\mathrm{Ee} / \mathrm{PAe}$ \\
\hline$N$-Z-L-Ser-L-leu-OH & klebrige Masse & - & - & - & - \\
\hline$N$-Z-L-Ser-L-ser-OH & $196-197,5$ & $\begin{array}{l}3 \\
4\end{array}$ & $\begin{array}{l}169-171 \\
186-187\end{array}$ & 69,6 & $\mathrm{Ee} / \mathrm{Dio} / \mathrm{PAe}$ \\
\hline$N$-Z-L-Ser-gly-gly-OH & $167,5-169,5$ & - & - & 35,1 & $\mathrm{Ee} / \mathrm{Dio} / \mathrm{PAe}$ \\
\hline
\end{tabular}

Tab. 1. N-Carbobenzoxy-aminosäuren und -peptidsäuren. Abkürzungen : Ee $=$ Essigester, Chl $=$ Chloroform, Dio $=$ Dioxan, $\mathrm{PAe}=$ Petroläther $\left(\mathrm{Sdp} .40-60{ }^{\circ} \mathrm{C}\right)$.

\begin{tabular}{|c|c|c|c|c|c|}
\hline Verbindung & $\begin{array}{l}\text { Schmp. } \\
{\left[{ }^{\circ} \mathrm{C}\right]}\end{array}$ & Literatur & $\begin{array}{c}\text { Lit.-Schmp. } \\
{\left[{ }^{\circ} \mathrm{C}\right]}\end{array}$ & $\begin{array}{c}\text { Ausbeute } \\
{[\%]}\end{array}$ & Umkrist. aus \\
\hline $\mathrm{H}-\mathrm{L}-\mathrm{Asp}(\mathrm{OBz})_{2} \cdot \mathrm{pts}$ & $161-162$ & 15 & $158-160$ & 80,0 & $\mathrm{Me} / \mathrm{Ae} / \mathrm{PAe}$ \\
\hline H-L-Glu $(\mathrm{OBz})_{2} \cdot \mathrm{pts}$ & $145-146$ & 15 & $144-145$ & 80,0 & $\mathrm{Me} / \mathrm{Ae} / \mathrm{PAe}$ \\
\hline H-Gly-OBz $\cdot$ pts & $132-135,5$ & 15 & $132-134$ & 89,0 & $\mathrm{Me} / \mathrm{Ae} / \mathrm{PAe}$ \\
\hline $\mathrm{H}$-L-Leu-OBz$\cdot \mathrm{pts}$ & $160-161$ & 15 & $158,5-160$ & 76,7 & $\mathrm{Me} / \mathrm{Ae} / \mathrm{PAe}$ \\
\hline $\mathrm{H}-\mathrm{L}-\mathrm{Phe}-\mathrm{OBz} \cdot \mathrm{pts}$ & $170-172$ & $\begin{array}{l}15 \\
40\end{array}$ & $\begin{array}{r}170,5-171,5 \\
164-165,5\end{array}$ & 70,6 & $\mathrm{Me} / \mathrm{Ae} / \mathrm{PAe}$ \\
\hline H-DL-Ser-OBz $\cdot$ pts & $106-108$ & - & - & 80,5 & $\mathrm{iP} / \mathrm{Ae}$ \\
\hline $\mathrm{H}-\mathrm{L}-\mathrm{Ser}-\mathrm{OBz} \cdot \mathrm{pts}$ & $65,5-68$ & 5 & $94-95$ & 76,8 & $\mathrm{iP} / \mathrm{Ae}$ \\
\hline H-L- $\alpha$-Ala-L-phe-OBz $\cdot$ pts & $137,5-139,5$ & - & - & 82,5 & $\mathrm{Me} / \mathrm{Ae} / \mathrm{PAe}$ \\
\hline H-Gly-gly-OBz $\cdot$ pts & $157,5-158,5$ & 18 & 153 & 84,7 & \\
\hline $\mathrm{H}$-Gly-L-ser-OBz $\cdot$ pts & oranges Öl & - & - & - & $\mathrm{iP} / \mathrm{Ae}$ \\
\hline H-L-Šer-gly-OBz $\cdot$ pts & $181-183$ & 32 & 180 & 65,9 & $\mathrm{Me} / \mathrm{Ae}$ \\
\hline H-Gly-gly-gly-gly-OBz $\cdot$ pts & $\begin{array}{l}196-199 \\
\text { (Zersetz.) }\end{array}$ & - & - & 33,6 & $\mathrm{DMF} / \mathrm{Ae}$ \\
\hline $\mathrm{H}$-Gly-gly-L-ser-OBz $\cdot \mathrm{HBr}$ & $95-98$ & - & - & 69,3 & $\mathrm{Me} / \mathrm{Ae} / \mathrm{PAe}$ \\
\hline
\end{tabular}

Tab. 2. Aminosäure- und Peptidbenzylester-p-toluolsulfonate bzw. -hydrobromide. Abkürzungen: A = Äthanol 95-proz., Ae $=$ Äther, $\mathrm{DMF}=$ Dimethylformamid, $\mathrm{iP}=$ iso-Propanol, $\mathrm{Me}=$ Methanol, $\mathrm{PAe}=$ Petroläther $\left(\mathrm{Sdp} .40-60{ }^{\circ} \mathrm{C}\right)$.

aufsatzes nach $\mathrm{Sta} \mathrm{rk}$ und $\mathrm{D}$ e a $\mathrm{n}$ fünf Stdn. lang azeotrop unter Rückfluß erhitzt. Danach wurde das Benzol und der überschüssige Benzylalkohol im Vakuum abdestilliert, wobei die Badtemperatur $125^{\circ} \mathrm{C}$ nicht überschritt. Zu dem Rückstand wurde absoluter Äther zugesetzt, bis keine Niederschlagsbildung mehr erfolgte.

32 D. Theodoropoulos, H. Bennich, G. Fölsch u. O. Mellander, Nature [London] 184, 187 [1959].

33 A. Winterstein, B. Hegedüs, B. Fust, E. Böhni u. A. Studer, Helv. chim. Acta 39, 229 [1956].

34 M. Bergmann u. L. Zervas, Ber. dtsch. chem. Ges. 65, 1192 [1932].

35 H. E. Carter, R. L. Frank u. M. W. Johnston, Org. Syntheses 23, 13 [1943].
Dann wurde das ganze mehrere Tage bei $0{ }^{\circ} \mathrm{C}$ aufbewahrt.

In der gleichen Weise wurden 4,93 g H-Gly-gly-gly. gly-OH mit $3,8 \mathrm{~g}$-Toluolsulfonsäure-monohydrat, $20 \mathrm{~cm}^{3}$ Benzylalkohol und $20 \mathrm{~cm}^{3}$ Benzol zur Reaktion gebracht.

36 R. A. Boissonnas u. G. Preitner, Helv. chim. Acta 36, 875 [1953].

37 E. Baer u. J. Maurukas, J. biol. Chemistry 212, 25 [1955].

38 J. A. Moore, J. R. Dice, E. D. Nicolaides, R. D. Westland u. L. Wittle, J. Amer. chem. Soc. 76, 2884 [1954].

39 S. Goldschmidt u. H. Lautenschlager, Liebigs Ann. Chem. 580, 68 [1953].

40 H. Schwarz u. K. Arakawa, J. Amer. chem. Soc. 81, 5691 [1959]. 


\section{L-a-Alanyl-L-phenylalanin-benzylester-p-toluolsulfonat}

Der entstandene Niederschlag wurde abgesaugt, mehrmals mit Äther gewaschen und an der Luft getrocknet. Das Rohprodukt wurde aus Methanol/ Äther unter Zusatz von Petroläther (Sdp. $40-60{ }^{\circ} \mathrm{C}$ ) umkristallisiert; kleine, weiße Nadeln.

Ausbeute: $10,29 \mathrm{~g} \triangleq 82,5 \%$ d. Th.; Schmp. 137,5 bis $139,5{ }^{\circ} \mathrm{C}$.

$\mathrm{C}_{26} \mathrm{H}_{30} \mathrm{~N}_{2} \mathrm{O}_{6} \mathrm{~S}(498,6)$

Ber. C 62,63 H 6,07 N 6,62 S 6,43,

Gef. C 61,98 H 6,14 N 5,51 S 6,41.

\section{Glycyl-glycin-benzylester-p-toluolsulfonat}

Der abgeschiedene Niederschlag wurde abgesaugt, mehrmals mit Äther gewaschen und an der Luft getrocknet. Zur Reinigung wurde aus 95-proz. Alkohol umkristallisiert; weiße, schuppenartige Kristalle.

Ausbeute: $8,35 \mathrm{~g} \leq 84,7 \%$ d. Th.; Schmp. 157,5 bis $158,5{ }^{\circ}$ C. Lit. l. c. ${ }^{18}$; Schmp. $153{ }^{\circ}$ C.

\section{Glycyl-L-serin-benzylester-p-toluolsulfonat}

Es hatte sich eine orange Schmiere an der Kolbenwandung niedergeschlagen. Die überstehenden Lösungsmittel wurden abdekantiert, der Rückstand wurde in Isopropanol gelöst, mit Äther erneut ausgefällt und bei $0{ }^{\circ} \mathrm{C}$ aufbewahrt. Das Produkt ließ sich nicht kristallisieren und wurde deshalb ohne weitere Reinigung für die nachfolgende Umsetzung eingesetzt.

Ausbeute: 11,3 g Rohprodukt.

$$
\text { L-Seryl-glycin-benzylester-p-toluolsulfonat }
$$

Ein aus weißen Flocken bestehender Niederschlag wurde abgesaugt und nach mehrmaligem Waschen mit Äther an der Luft getrocknet. Rohprodukt 7,47 g,
Schmp. $180,5-184{ }^{\circ} \mathrm{C}$. Das Rohprodukt wurde aus Methanol/ ̈̈ther umkristallisiert; weiße, schuppenartige Kristalle.

Ausbeute: $6,98 \mathrm{~g} \triangleq 65,9 \%$ d. Th.; Schmp. 181 bis $183^{\circ}$ C. Lit. 1. c. ${ }^{32}$; Schmp. $180^{\circ}$ C.

\section{Glycyl-glycyl-glycyl-glycin-benzylester-p-toluolsulfonat}

Es hatten sich orange Flocken abgesetzt, die abgesaugt und mehrmals mit absolutem Äther gewaschen wurden. Nach dem Trocknen an der Luft wurde das Rohprodukt aus DMF/Äther umkristallisiert; weißes Pulver.

Ausbeute: $3,42 \mathrm{~g} \triangleq 33,6 \%$ d. Th.; Schmp. 196 bis $199^{\circ} \mathrm{C}$, Zersetzung.

$\mathrm{C}_{22} \mathrm{H}_{28} \mathrm{~N}_{4} \mathrm{O}_{8} \mathrm{~S}(508,5)$

$$
\begin{aligned}
& \text { Ber. C } 51,96 \text { H } 5,55 \text { N } 11,02 \text { S 6,30, } \\
& \text { Gef. C } 52,08 \text { H } 5,73 \text { N 10,84 S 6,42. }
\end{aligned}
$$

$$
\text { Glycyl-glycyl-L-serin-benzylester-hydrobromid }
$$

$2,22 \mathrm{~g} \mathrm{~N}$-Z-Gly-gly-L-ser-OBz wurden in $50 \mathrm{~cm}^{3}$ Nitromethan suspendiert und unter starkem Rühren 30 Min. lang ein lebhafter Gasstrom von $\mathrm{HBr}^{*}$ in die Suspension eingeleitet. Danach wurde die Suspension sofort mit $150 \mathrm{~cm}^{3}$ absolutem Äther versetzt und drei Stdn. bei $-15{ }^{\circ} \mathrm{C}$ aufbewahrt. Die Lösungsmittel wurden nun vom Niederschlag abdekantiert, der Niederschlag zweimal mit je $20 \mathrm{~cm}^{3}$ absolutem Äther versetzt und nach dem Umschütteln das Lösungsmittel jedesmal erneut abdekantiert. Der Rückstand wurde aus Methanol/Äther unter Zusatz von Petroläther (Sdp. $40-60^{\circ} \mathrm{C}$ ) umkristallisiert; weißes Pulver.

Ausbeute: $1,35 \mathrm{~g} \triangleq 69,3 \%$ d. Th.; Schmp. $95-98{ }^{\circ} \mathrm{C}$. $\mathrm{C}_{14} \mathrm{H}_{20} \mathrm{~N}_{3} \mathrm{O}_{5} \mathrm{Br}(390,2)$

Ber. C 43,09 H 5,17 N 10,77 Br 20,48,

Gef. C 40,65 H 5,86 N 10,46 Br 21,01.

* Der Firma Schering AG in Berlin danke ich für die freundliche Überlassung einer $\mathrm{HBr}$-Bombe. 